\title{
Riesgo suicida de acuerdo al tratamiento farmacológico para la cesación tabáquica
}

\author{
Suicidal risk according pharmacological smoking cessation therapy
}

Gunnell, D y col. BMJ 2009; 339:b3805

\section{Objetivo}

Determinar si el tratamiento con varenicline para la cesación tabáquica, se asocia a un aumento del riesgo de suicidio y/o de la conducta suicida comparado con bupropión y con la terapia de reemplazo nicotínica (TRN).

\section{Diseño, lugar, participantes y resultados evaluados}

Cohorte anidada* en una base de datos de investigación en Atención Primaria del Reino Unido (UK) que registra datos demográficos, consultas, prescripciones y resultados relacionados con la salud de 3,6 millones de pacientes.

Fueron identificados 80660 individuos de 18 a 95 años de edad a quienes se les había prescripto tratamiento para dejar de fumar entre 2006 y 2008 y tenían al menos un año de registro en el sistema. La fecha de la primera prescripción definía el ingreso a la cohorte, extendiéndose el seguimiento hasta tres meses después de la fecha de la última prescripción. Los pacientes fueron asignados al grupo de la primera droga prescripta desde el ingreso a esta cohorte: 1) varenicline ( $n=10973) ; 2)$ bupropión $(n=6422) ; 3)$ terapia de reemplazo nicotínico $(n=63625)$.

Los resultados primarios fueron suicidio o intento de suicidio (contándose con un poder de $80 \%$ para detectar un aumento del doble de su incidencia) y los secundarios, la de pensamientos suicidas y depresión (definida por la prescripción de tratamiento antidepresivo).

\section{Resultados}

Los antecedentes de uso de psicofármacos, de consumo inadecuado de alcohol, del número de consultas psiquiátricas y de intentos de suicidios previos al inicio de esta cohorte fueron más prevalentes en los pacientes a quienes se les prescribió TRN.

Fueron registradas 168 lesiones autoprovocadas, 166 intentos de suicidio y dos suicidios.

Tomando como referencia al grupo asignado a TRN y luego de ajustar por los potenciales confundidores* se observó una tendencia estadísticamente no significativa de mayor riesgo de autolesiones para los tratados con bupropión (RR 1,7; IC95 0,59 a 2,32) y con varenicline (RR 1,12; IC95 0,67 a 1,88). No hubo evidencia de que varenicline se asociase con un aumento del riesgo de depresión ( $\mathrm{n}=2242 ; \mathrm{RR} 0,88 ;$ IC95; 0,77 a 1,00) ó de mayor reporte de ideación suicida ( $n=37$; RR 1,43; IC95 0,53 a 3,85).

\section{Conclusión}

No se pudo establecer asociación entre el uso de Varenicline y el aumento al doble del riesgo de suicidio, pero no puede ser descartado.

Palabras claves: Varenicline, Bupropión, Terapia de Reemplazo Nicotinica, Conducta suicida.

Keywords: Varenicline, Bupropión, Nicotine Replacement Products, Suicidal Behaviour.

Fuentes de financiamiento: Medicines and Healthcare Products Regulatory Agency (MHRA)

\section{Comentario}

El tabaquismo provoca una de cada diez muertes durante la edad adulta, siendo la principal causa de mortalidad evitable ${ }^{1}$. Diferentes teorías asocian el consumo de tabaco con un aumento del riesgo de suicidio, debido a su efecto tóxico directo, y a su uso como estimulante por personas deprimidas y abusadoras de alcohol (a su vez, factores de riesgo de suicidio) entre otras ${ }^{2}$. El suicidio es un fenómeno complejo e involucra factores biológicos, psicológicos y socio-culturales, estimándose que cada año muere un millón de personas en el mundo por esta causa y que al menos 20 personas lo intentan por cada una que lo concreta ${ }^{3}$. En este estudio esa relación fue mayor, lo que incitaría a pensar que pudo haber casos de suicidio no identificados. Además, la baja cantidad de registro de pensamientos suicidas en relación a la de los intentos, puede implicar un subregistro de aquellos. Vale destacar que este estudio contó con un poder del $80 \%$ para detectar un aumento al doble en la incidencia de suicidio, pudiendo haber pasado inadvertido un aumento menor, pero quizás clínicamente relevante.

\section{Conclusión del comentador}

Por su efectividad, varenicline es considerada una droga de primera línea ${ }^{4}$. Sin embargo y como en toda decisión médica, la evaluación de los beneficios y los riesgos de la intervención deben ser considerados ante cada paciente. No debemos olvidar que cualquier intervención realizada en el consultorio debe ser acompañada de estrategias dirigidas a la población en general, siendo en tabaquismo las políticas públicas las más efectivas en relación con los costos. En 2003 la Organización Mundial de la Salud adoptó por unanimidad el Convenio Marco para el Control del Tabaco ${ }^{5}$-primer tratado mundial de salud pública y primer instrumento jurídico diseñado para reducir la morbimortalidad relacionada con el tabaquismo. Su objetivo es proteger a las generaciones actuales y futuras de las graves consecuencias sanitarias, socio-ambientales y económicas que acarrea el consumo del tabaco. A menos que se apliquen eficazmente las disposiciones del tratado, las muertes relacionadas con el tabaco podrían superar los ocho millones para 2030'. Lamentablemente Argentina es uno de los países que aun no lo ha ratificado.

Ver glosario*

Paula Chávez [ Equipo Psy. Fundación Equidad. Chavezpaula69@gmail.com ]

Chávez P. Riesgo suicida de acuerdo al tratamiento farmacológico para la cesación tabáquica. Evid Act Pract Ambul. 13(3). 85. Jul-Sept 2010. Comentado de: Varenicline and suicidal behaviour: a cohort study based on data from the General Practice Research Database. Gunnell, D y col. BMJ 2009; 339:b3805. PMID: 19797344.

\section{Referencias}

1. World Health Organization. WHO report on the global tobacco epidemic, 2009: implementing smoke-free environments.

Disponible en http://www.who.int/tobacco/mpower/2009/gtcr_download/en/index.html (último acceso: 13/08/10).

2. Hughes JR. Smoking and suicide: a brief overview. Drug Alcohol Depend. 2008 December 1; 98 (3): 169-178.
3. World Health Organization. Suicide prevention (SUPRE). Disponible en http://www.who.int/mental health/prevention/suicide/suicideprevent (último acceso: 13/08/10). 3. World Health Organization. Suicide prevention (SUPRE). Disponible en http://www.who.int/mental_health/prevention/suicide/suicideprevent (último acceso: $13 / 08 / 10)$.
4. Fiore MC, Jaén CR, Baker TB, et al. Treating Tobacco Use and Dependence:2008 Update. Clinical Practice Guideline. Rockville, MD: U.S. Department of Health and Human Services.

5. 56 ${ }^{\mathrm{a}}$ Asamblea Mundial de la Salud. Convenio Marco de la OMS para el Control del Tabaco.2003. Ginebra. Organización Mundial de la Salud.

Disponible en: http://www.paho.org/Spanish/DD/PUB/sa56r1.pdf (último acceso: 13/08/10). 